\title{
Polychaetes from Burdwood Bank: "Namuncurá I" Marine Protected Area and slope, SW Atlantic Ocean
}

\author{
Claudia Bremec ${ }^{1}$, Rodolfo Elías ${ }^{2}$, Sofía Calla ${ }^{3}$, Gabriel Genzano $^{1}$, Alejandro Puente Tapia ${ }^{1}$ \& \\ Laura Schejter ${ }^{4}$
}

1. Estación Costera J.J. Nagera, Universidad Nacional de Mar del Plata. Funes 3350, 7600 Mar del Plata, Argentina. Instituto de Investigaciones Marinas y Costeras, Consejo Nacional de Investigaciones Científicas y Técnicas (IIMyCCONICET), Argentina; claudiasilviabremec@gmail.com

2. Facultad de Ciencias Exactas y Naturales, Universidad Nacional de Mar del Plata, Deán Funes 3350, B7602AYL Mar del Plata, Argentina; roelias@mdp.edu.ar

3. Museo Argentino de Ciencias Naturales «Bernardino Rivadavia”, Av. Ángel Gallardo 470, C1405DJR Buenos Aires, Argentina. Consejo Nacional de Investigaciones Científicas y Técnicas (CONICET); sofialcalla@gmail.com

4. Instituto Nacional de Investigación y Desarrollo Pesquero (INIDEP). Paseo Victoria Ocampo Nº1, 7600, Mar del Plata, Argentina. Instituto de Investigaciones Marinas y Costeras, Consejo Nacional de Investigaciones Científicas y Técnicas (IIMyC- CONICET), Argentina; corresponding author, schejter@inidep.edu.ar

\section{Received 30-XI-2018. C Corrected 03-V-2019. Accepted 30-VI-2019.}

\begin{abstract}
Introduction: The first open-sea (non-coastal) Marine Protected Area in Argentina, named "Namuncurá I" (NMPA), was created in 2013 at Burdwood Bank (BB), an undersea plateau located about $200 \mathrm{~km}$ south from Malvinas Islands (Falkland Islands) and $150 \mathrm{~km}$ east from Staten Island, SW Atlantic Ocean. It comprises three different management areas: the central one ("core", strict protection, only control and monitoring activities), surrounded by a "buffer" area (authorized activities, e.g. scientific research) and an external "transition" area (productive and extractive activities contemplated in the Management Plan). Beyond the transition area, only the southern shelf-break is protected, after the creation of the "Namuncurá II" MPA in December 2018. Objective: provide the inventory of polychaetes collected during 2016 and 2017 at the core (98 m depth), buffer (128 m depth), transition (133 m-189 m depth) areas of the Namuncurá I MPA, together with slope areas (220 m-798 m depth). Methods: taken with a trawl net used to characterize the faunal assemblages and to compare results with other Magellan areas. Multidimensional Scaling (MDS) and Cluster Analysis (CA) were applied to a Bray-Curtis similarity index to assess polychaete assemblages in the NMPA BB slope and Magellan region respectively; SIMPER (Similarity Percentage Analyses) and ANOSIM (Analysis of Similarities) were performed. Results: A total of 918 individuals, which correspond to 39 taxa distributed in 22 families, were recorded in samples from NMPA and BB slope, mainly epibenthic or associated with the large corals collected. Ampharete kerguelensis McIntosh, 1885; Hyalinoecia artifex Verrill, 1880; Idanthyrsus macropaleus (Schmarda, 1861); Laetmonice producta Grube, 1877; Onuphis pseudoiridescens Averincev, 1972; Pista mirabilis McIntosh 1885 and Terebellides malvinensis Bremec \& Elias, 1999 constitute new records for Burdwood Bank. A similar polychaete assemblage characterized the three areas of NMPA and slope locations, while $H$. artifex characterized locations at the NW deepest slope of the bank. Conclusions: These results indicate strong connections between the fauna collected at NMPA and the polychaetes assemblage in other Magellan areas dominated by soft bottoms. The biogeographic importance of the BB as connection for benthic polychaete species between South America and the Antarctic Peninsula merits future investigation.
\end{abstract}

Key words: new Marine Protected Area, Burdwood Bank, polychaetes, SW Atlantic Ocean.

Bremec, C., Elías, R., Calla, S., Genzano G., Puente Tapia, A., \& Schejter, L. (2019). Polychaetes from Burdwood Bank: "Namuncurá I" Marine Protected Area and slope. Revista de Biología Tropical, 67(S5) Suplemento, S119-S135. 
Most of the research on polychaetes (annelids) collected in southern regions refers to subantarctic species from the Patagonian shelf, the Strait of Magellan and Antarctica collected during international exploratory cruises (Blake, 1983; Bremec \& Elías, 1999; Gambi \& Mariani, 1999; Hartman, 1953, 1966; Hartmann-Schroeder, 1983; Hartmann-Schroeder \& Hartmann, 1962; Lana \& Bremec, 1994; Mariani, Gambi, Lorenti, \& Mazzella, 1996; Montiel, Gerdes, Hilbig, \& Arntz, 2005, Montiel, Gerdes, \& Arntz, 2005; Montiel, Quiroga, \& Gerdes, 2011; Orensanz, 1974, 1990; Rozbaczylo, Ríos, \& Mutschke, 1997; Sanfilippo, 1994; Uschakov, 1962; Wesenberg-Lund, 1962; Bremec, Elías, \& Gambi, 2000; Bremec, Souto, \& Genzano, 2010; Elías, Bremec, Lana, \& Orensanz, 2003). In particular at Burdwood Bank (BB), a few locations were sampled in the last decades during "Walther Herwig" (1978, stations 595 and 596; Hartmann-Schroeder, 1983), "Shinkai Maru" (1978-1979, stations 133, 135 and 138; Bremec et al., 2010) and "Polarstern" (2002, stations 145 and 150; Montiel et al., 2003) cruises. The plateau represents one of the "stepping stones" linking the southern tip of South America with the Antarctic Peninsula (Thomson, 2004; Arntz, Lovrich, \& Thatje, 2005; Griffiths, Linse, \& Barnes, 2008) and, in the case of polychaetes, faunal relationships between the Magellan region and the Antarctic continent were not established. Apart from a single taxon, orbiniid species found in the Southern Ocean are effectively isolated from South American species (Blake, 2017). It was suggested that comparable studies are needed to investigate affinities between both neighboring ecosystems, separated only by the Drake Passage and the Antarctic Convergence (Cañete, Leighton, \& Aguilera, 1999; Montiel, Gerdes, Hilbig et al., 2005; Montiel, Gerdes et al., 2005).

BB is a segment of the North Scotia Ridge in the SW Atlantic Ocean, located between $54^{\circ}-55^{\circ} \mathrm{S}$ and $56^{\circ}-62^{\circ} \mathrm{W}$. This undersea plateau is located about $200 \mathrm{~km}$ south from Malvinas Islands (Falkland Islands) and about 150 $\mathrm{km}$ east from Staten Island, Tierra del Fuego
Province. The rocky slopes of the bank rise from more than $4000 \mathrm{~m}$ depth in the Yaghan Basin (Drake Passage) to form a wide plateau with depths that vary between $50 \mathrm{~m}$ and $200 \mathrm{~m}$, where the bottom abruptly breaks into a wall reaching $1100 \mathrm{~m}$ to more than 3000 $\mathrm{m}$ depth. The direct influence of the Antarctic Circumpolar Current and persistent westerlies blowing at $50^{\circ} \mathrm{S}$ make the region an extremely dynamic environment of subantarctic water, fairly homogeneous vertically in the plateau (without a thermocline), ranging seasonally from 4 to $9{ }^{\circ} \mathrm{C}$ and with a mean salinity of 34 (Piola \& Gordon, 1989; Guerrero, Baldoni, \& Benavides, 1999). BB is surrounded by the Malvinas Current, a branch of the Circumpolar Antarctic Current that flows from the Drake Passage, which is one of the most important and nutrient rich currents of the sea (Piola \& Gordon, 1989; Lutz et al., 2010). Although the existence of BB has been known since 1842 (Findlay, 1867), the benthic realm has been only scarcely studied.

The first open-sea (non-coastal) Marine Protected Area in Argentina, named "Namuncurá I" (NMPA, National Law 26875, Argentina), was created in 2013 at Burdwood Bank, Argentine Sea, and comprises nearly $28000 \mathrm{~km}^{2}$ circumscribed by the $200 \mathrm{~m}$ isobath. It comprises three different management areas according to the protection level required: the central "core" (strict protection, only control and monitoring activities), surrounded by a "buffer" area (authorized activities, e.g. scientific research) and an external "transition" area (productive and extractive activities contemplated in the Management Plan). Beyond the transition area, only the southern shelf-break is protected since December 2018, when the "Namuncurá II" MPA was created (National Law 27490, Argentina). After recent research cruises, Schejter et al. (2016) preliminary recorded nearly 250 species of epibenthic organisms, and particular studies on Peracarida (Doti et al., 2014; Chiesa, Urteaga, Martínez, Doti, \& Roccatagliata, 2015), Cnidaria (Cairns, 2012), Porifera (Schejter, Bertolino, \& 
Calcinai, 2017) and Asteroidea (Fraysse, Calcagno, \& Pérez, 2018) were developed.

The present objectives are to provide the inventory of polychaetes collected at the core (98 $\mathrm{m}$ depth), buffer (128 $\mathrm{m}$ depth) and transition (133-189 m depth) areas of NMPA and BB slope (220-798 m depth), where intensive sampling with trawls was performed to characterize the faunal assemblages and to explore affinities of the polychaete fauna between BB and other Magellanic areas.

\section{MATERIALS AND METHODS}

The NMPA and BB slope (S) were sampled during three research cruises onboard the GC-189 "Pr. García" (December 2015) and RV "Puerto Deseado" (March-April 2016; April-May 2017). Samples were taken in 30 stations (Fig. 1, Table 1) with bottom trawls of $10 \mathrm{~mm}$ mesh size. Faunal comparison with other Magellan areas was made using presenceabsence data (due to the different sampling

TABLE 1

Geographical position, depth and cruise information of the stations sampled at Burdwood Bank Slope (BBS), Transition (MPA-T), Buffer (MPA-B) and Core (MPA-C) areas during cruises "Puerto Deseado 2016" (BBB 16), "Puerto Deseado 2017" (PD BB 17) and "GC Pr. García 2015" (GC 15)

\begin{tabular}{|c|c|c|c|c|c|c|c|c|}
\hline Site & Cruise & Date $(\mathrm{d} / \mathrm{m} / \mathrm{y})$ & Station & Haul & Sub-area & Lat. ${ }^{\circ} \mathrm{S}$ & Long. ${ }^{\circ} \mathrm{W}$ & Depth (m) \\
\hline $\mathrm{A}$ & BBB16 & $13 / 04 / 2016$ & 18 & 266 & BBS & -54.8209833 & -60.7035833 & 607 \\
\hline B & BBB16 & $13 / 04 / 2016$ & 21 & 239 & BBS & -54.8869667 & -59.815 & 785 \\
\hline $\mathrm{C}$ & BBB16 & $13 / 04 / 2016$ & 23 & 226 & MPA-T & -54.7596 & -59.8689167 & 182 \\
\hline $\mathrm{D}$ & BBB16 & $29 / 03 / 2016$ & 26 & 27 & MPA-T & -54.4158 & -58.5151667 & 137 \\
\hline E & BBB16 & $28 / 03 / 2016$ & 27 & 11 & MPA-T & -54.16775 & -58.2726167 & 100 \\
\hline $\mathrm{F}$ & BBB16 & $30 / 03 / 2016$ & 28 & 52 & MPA-B & -54.4593833 & -59.2201667 & 128 \\
\hline G & BBB16 & $10 / 04 / 2016$ & 30 & 184 & MPA-C & -54.2884833 & -59.9507833 & 96 \\
\hline $\mathrm{H}$ & BBB16 & $10 / 04 / 2016$ & 31 & 197 & MPA-C & -54.4993667 & -59.8588667 & 109 \\
\hline I & BBB16 & $30 / 03 / 2016$ & 32 & 77 & MPA-C & -54.5433167 & -60.0213167 & 98 \\
\hline $\mathrm{J}$ & BBB16 & $8 / 04 / 2016$ & 33 & 159 & MPA-B & -54.4295333 & -60.6477167 & 101 \\
\hline $\mathrm{K}$ & BBB16 & $7 / 04 / 2016$ & 34 & 146 & MPA-B & -54.4543 & -60.9803333 & 100 \\
\hline $\mathrm{L}$ & BBB16 & $31 / 03 / 2016$ & 35 & 89 & MPA-T & -54.5319833 & -61.4385167 & 125 \\
\hline M & BBB16 & $19 / 04 / 2016$ & 36 & 306 & MPA-T & -53.9298833 & -61.4956 & 185 \\
\hline $\mathrm{N}$ & BBB16 & $29 / 04 / 2016$ & 38 & 39 & MPA-T & -54.5887333 & -58.5472167 & 135 \\
\hline $\mathrm{O}$ & BBB16 & $19 / 04 / 2016$ & 40 & 320 & BBS & -54.6167667 & -61.4208333 & 415 \\
\hline$P$ & PD BB 17 & $28 / 04 / 2017$ & 16 & 131 & BBS & -54.600012 & -61.5100102 & 294 \\
\hline Q & PD BB 17 & $1 / 05 / 2017$ & 23 & 173 & MPA-C & -54.43342 & -59.5033434 & 91 \\
\hline $\mathrm{R}$ & PD BB 17 & $1 / 05 / 2017$ & 24 & 184 & MPA-C & -54.33334 & -59.8950179 & 97 \\
\hline S & PD BB 17 & $9 / 05 / 2017$ & 25 & 304 & MPA-C & -54.3450069 & -60.3450069 & 104 \\
\hline $\mathrm{T}$ & PD BB 17 & $9 / 05 / 2017$ & 26 & 317 & MPA-B & -54.100002 & -60.7100142 & 120 \\
\hline $\mathrm{U}$ & PD BB 17 & $9 / 05 / 2017$ & 27 & 326 & MPA-B & -54.1066688 & -60.8783509 & 128 \\
\hline V & PD BB 17 & $8 / 05 / 2017$ & 29 & 283 & MPA-T & -53.8150163 & -61.3200064 & 197 \\
\hline W & PD BB 17 & $8 / 05 / 2017$ & 30 & 273 & BBS & -53.8216831 & -61.4733428 & 209 \\
\hline $\mathrm{X}$ & PD BB 17 & $7 / 05 / 2017$ & 31 & 269 & BBS & -53.66668 & -61.6366794 & 642 \\
\hline $\mathrm{Y}$ & PD BB 17 & $7 / 05 / 2017$ & 33 & 256 & BBS & -53.466676 & -61.8416835 & 595 \\
\hline $\mathrm{Z}$ & PD BB 17 & $30 / 04 / 2017$ & 21 & 157 & MPA-T & -54.4250085 & -58.5250105 & 138 \\
\hline $\mathrm{A}^{\prime}$ & GC 15 & $12 / 12 / 2015$ & 14 & $8 \mathrm{~b}$ & MPA-C & -54.439467 & -60.652217 & 99 \\
\hline $\mathrm{B}^{\prime}$ & GC 15 & $13 / 12 / 2015$ & 17 & 1 & MPA-T & -54.4855 & -59.081217 & 138 \\
\hline $\mathrm{C}^{\prime}$ & GC 15 & $13 / 12 / 2015$ & $17 b$ & 2 & MPA-T & -54.45215 & -59.1285 & 138 \\
\hline $\mathrm{D}^{\prime}$ & PD BB 17 & $8 / 05 / 2017$ & 28 & 287 & MPA-T & -54.0533344 & -61.0950019 & 140 \\
\hline
\end{tabular}




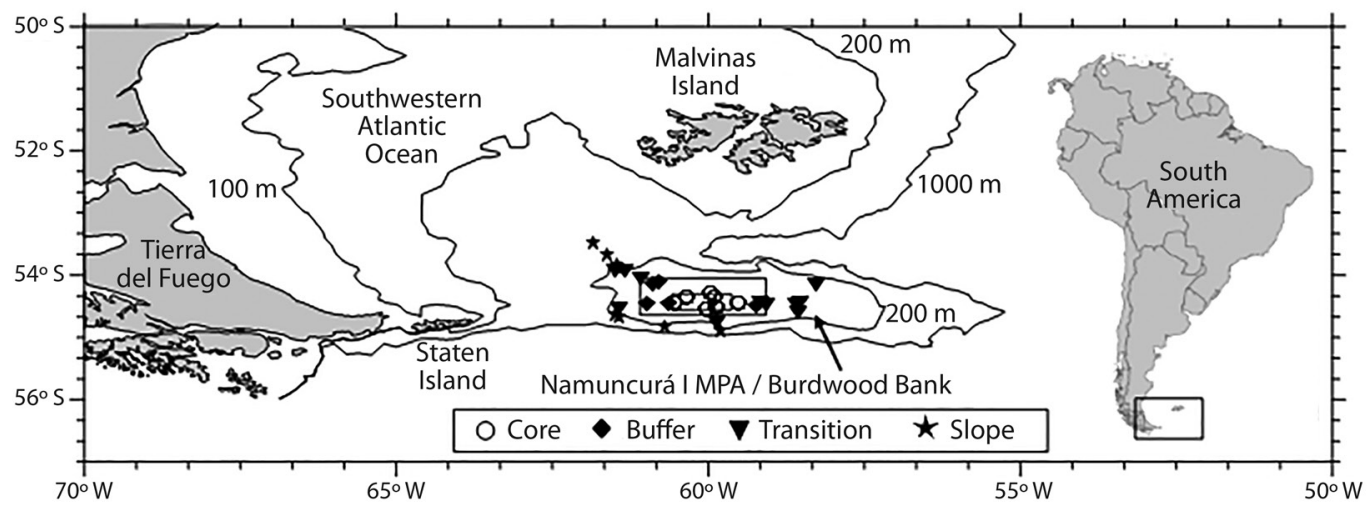

Fig. 1. Location of the sampling sites at Burdwood Bank in the different areas of Namuncurá Marine Protected Area and slope.

tools employed) from neighboring areas $(\mathrm{N})$, Staten Island and coastal waters of Tierra del Fuego (TDF), and the Patagonian shelf (PS) (Table 2). Samples from N were collected during the "Puerto Deseado" 2017 expedition. Samples collected during "GC-189 Pr. García" 2015 and used for this regional comparison were collected with bottom trawls of $2 \mathrm{~mm}$ mesh size (Güller, Abelando, Urcola, \& Zelaya, 2015). Other additional polychaete data come from "Shinkai Maru" cruises IV, V, X and XI, collected with Picard dredge from soft bottoms and sieved with $1 \mathrm{~mm}$ mesh screen (Bremec et al., 2010). Specimens collected during "Puerto Deseado" 2016-2017 and "GC-189 Pr. García" 2015 cruises were fixed in $4 \%$ formaldehyde, preserved in alcohol $70 \%$ and identified at the lowest taxonomic level possible. Taxonomic determinations were made based on Hartman, 1953, 1966; Wesenberg-Lund, 1962; Blake, 1983; Lana \& Bremec, 1994; Bremec \& Elías, 1999; Boggemann \& Orenzanz, 2007; Barnich, Orensanz, \& Fiege, 2012. Taxonomy and distribution were matched against the Register of Antarctic Marine Species (Clarke \& Johnston, 2003), Bremec \& Giberto (2008), Orensanz, Diez, Ferrando, \& Trovant (2012), World Register of Marine Species (Horton et al., 2018) and Ocean biogeographic information system [OBIS] (2018) to ensure that synonymies or misspellings were removed, as well as to compare the known distribution to that recorded in this study.

Multidimensional Scaling (MDS) and Cluster Analysis (CA) were applied to a BrayCurtis similarity index to assess polychaete assemblages in the NMPA - BB slope and Magellan region respectively. Similarity Percentage Analyses (SIMPER) was applied to describe the contribution of species to the dissimilarity between groups of stations. An Analysis of Similarities (ANOSIM) was carried out between samples located in the NMPA and BB slope to assess polychaete assemblages inside and outside the NMPA, and between samples from NMPA, S, N, TDF and PS to analyze polychaete distribution patterns in the Magellan region, considering the null hypothesis of no differences between areas. We used PRIMER version 6.1 (Clarke \& Gorley, 2006) with presence-absence data, excluding unique findings.

Voucher specimens of the polychaetes collected during recent cruises were deposited at the Museo Argentino de Ciencias Naturales "Bernardino Rivadavia", Buenos Aires, Argentina.

\section{RESULTS}

A total of 918 individuals, which correspond to 39 taxa distributed in 22 families, 
TABLE 2

Geographical position and depth of the stations sampled at Burdwood Bank Neighboring locations (N),

Tierra del Fuego (TDF), Patagonian Shelf (PS) and Namuncurá Marine Protected Area (MPA) during

"Puerto Deseado 2017" (PD), "GC Pr. García 2015" (GC) and "Shinkai Maru 1978-1979" (SM) cruises

\begin{tabular}{|c|c|c|c|c|c|}
\hline Cruise & Station & Area & Lat ${ }^{\circ} \mathrm{S}$ & Long. ${ }^{\circ} \mathrm{W}$ & Depth (m) \\
\hline PD & 34 & $\mathrm{~N}$ & -53.5680114 & -62.9656026 & 516 \\
\hline PD & 35 & $\mathrm{~N}$ & -53.5747782 & -63.9987033 & 236 \\
\hline PD & 36 & $\mathrm{~N}$ & -53.7297479 & -64.5076435 & 137 \\
\hline $\mathrm{GC}$ & 1 & TDF & -54.894583 & -67.675783 & 75 \\
\hline $\mathrm{GC}$ & 2 & TDF & -54.9124 & -67.235733 & 38 \\
\hline $\mathrm{GC}$ & 3 & TDF & -54.958333 & -66.919817 & 64 \\
\hline $\mathrm{GC}$ & 4 & TDF & -55.065433 & -66.68285 & 38 \\
\hline GC & $4 B$ & TDF & -55.09805 & -66.3373 & 45 \\
\hline $\mathrm{GC}$ & 5 & TDF & -55.041333 & -66.074417 & 86 \\
\hline $\mathrm{GC}$ & 6 & TDF & -55.006767 & -65.828 & 103 \\
\hline GC & $6 \mathrm{~B}$ & TDF & -54.956267 & -65.4572 & 56 \\
\hline GC & FH & TDF & -54.8965 & -67.313883 & 25 \\
\hline SM V & 36 & PS & -43.4504234 & -63.4682603 & 72 \\
\hline SM V & 37 & PS & -43.51677 & -61.9843635 & 91 \\
\hline SM X & 40 & PS & -43.45009 & -59.53344 & 145 \\
\hline SM X & 44 & PS & -44.5001 & -62.48343 & 103 \\
\hline SM IV & 55 & PS & -46.55011 & -65.5171034 & 79 \\
\hline SM XI & 55 & PS & -46.51677 & -65.45009 & 72 \\
\hline SM XI & 57 & PS & -46.5001 & -63.43342 & 115 \\
\hline SM X & 59 & PS & -46.46676 & -61.5001 & 121 \\
\hline SM IV & 60 & PS & -46.48343 & -60.46676 & 155 \\
\hline SM XI & 78 & PS & -49.48343 & -64.48343 & 120 \\
\hline SM V & 79 & PS & -49.4505901 & -62.4675935 & 152 \\
\hline SM V & 81 & PS & -49.5001 & -60.5337734 & 178 \\
\hline SM XI & 82 & PS & -49.46676 & -60.46676 & 188 \\
\hline SM IV & 89 & PS & -50.483343 & -65.50001 & 117 \\
\hline SM XI & 92 & PS & -50.450009 & -63.583345 & 154 \\
\hline SM IV & 93 & PS & -50.516677 & -60.483343 & 154 \\
\hline SM X & 95 & PS & -50.483343 & -59.483343 & 152 \\
\hline SM IV & 96 & PS & -50.533344 & -57.933352 & 143 \\
\hline SM V & 99 & PS & -51.5339401 & -67.5842835 & 100 \\
\hline SM V & 101 & PS & -51.4847636 & -65.5337734 & 134 \\
\hline SM V & 102 & PS & -51.5014336 & -63.3175635 & 180 \\
\hline SM XI & 101 & PS & -51.40008 & -65.48343 & 135 \\
\hline SM XI & 105 & PS & -51.48343 & -61.8335 & 192 \\
\hline SM X & 106 & PS & -51.63346 & -57.30006 & 189 \\
\hline SM IV & 108 & PS & -52.516677 & -67.300006 & 92 \\
\hline SM IV & 111 & PS & -52.483343 & -64.583345 & 183 \\
\hline SM XI & 111 & PS & -52.616679 & -65.516677 & 125 \\
\hline SM IV & 119 & PS & -53.516677 & -66.450009 & 95 \\
\hline SM XI & 120 & PS & -53.416675 & -66.550011 & 92 \\
\hline SM X & 122 & PS & -53.300006 & -64.416675 & 169 \\
\hline SM IV & 128 & TDF & -54.5001 & -64.41675 & 111 \\
\hline SM X & 128 & PS & -54.33334 & -65.466676 & 93 \\
\hline SM X & 133 & MPA & -54.26672 & -60.05001 & 100 \\
\hline SM X & 135 & MPA & -54.5001 & -58.5001 & 133 \\
\hline SM X & 138 & MPA & -54.5001 & -56.58345 & 135 \\
\hline SM V & $\mathrm{Ad} 3$ & PS & -49.4177502 & -63.4344202 & 145 \\
\hline SM XI & $\mathrm{Ad} 3$ & PS & -44.56678 & -65.01667 & 82 \\
\hline SM XI & Ad5 & PS & -47.06668 & -65.45009 & 70 \\
\hline SM XI & Ad11 & TDF & -54.21671 & -66.55011 & 55 \\
\hline SM XI & $\operatorname{Ad} 14$ & PS & -43.55011 & -59.8335 & 116 \\
\hline
\end{tabular}


were recorded in samples from NMPA and BB slope (Table 3). Terebellidae and Polynoidae were the most diverse families, represented by five taxa each. Polyeunoa laevis McIntosh,
1885 and Serpula narconensis Baird, 1865 were most frequently collected in the different zones of NMPA and BB slope. Nicolea chilensis (Schmarda, 1861), Trypanosyllis gigantea

TABLE 3

Polychaete taxa recorded at the sites (see Table 1) sampled at Burdwood Bank Slope (BB-Slope), Transition (MPATransition), Buffer (MPA-Buffer) and Core (MPA-Core) areas during the cruises and sites referred in Table 1

\begin{tabular}{|c|c|c|c|c|c|}
\hline Taxa recorded & MPA-Core & MPA-Buffer & MPA-Transition & BB-Slope & $\mathrm{N}^{\circ}$ Sites \\
\hline Aglaophamus virginis (Kinberg, 1866) & $\mathrm{A}^{\prime}$ & & E, $\mathrm{B}^{\prime}$ & & 3 \\
\hline Ampharete kerguelensis Mclntosh, 1885 & & & $\mathrm{~B}^{\prime}$ & & 1 \\
\hline Anobothrus sp. & & & $\mathrm{C}$ & & 1 \\
\hline Boccardia sp. & $\mathrm{S}$ & & $\mathrm{D}^{\prime}, \mathrm{V}$ & & 3 \\
\hline Chaetopterus antartcticus Kinberg, 1866 & $\mathrm{H}, \mathrm{I}, \mathrm{Q}, \mathrm{R}$ & $\mathrm{F}$ & & & 5 \\
\hline Eteone aurantiaca Schmarda, 1861 & $\mathrm{H}, \mathrm{Q}, \mathrm{R}, \mathrm{S}$ & U & $\mathrm{D}^{\prime}, \mathrm{T}$ & & 7 \\
\hline Eucranta sp. & $\mathrm{Q}, \mathrm{R}, \mathrm{S}$ & $\mathrm{J}, \mathrm{K}, \mathrm{T}, \mathrm{U}$ & $\mathrm{C}, \mathrm{D}, \mathrm{E}, \mathrm{M}, \mathrm{D}^{\prime}$ & & 12 \\
\hline Eunereis patagonica (Mclntosh, 1885) & $\mathrm{Q}, \mathrm{R}, \mathrm{A}^{\prime}$ & $\mathrm{J}, \mathrm{K}, \mathrm{T}, \mathrm{U}$ & $\mathrm{E}, \mathrm{M}, \mathrm{N}, \mathrm{D}^{\prime}, \mathrm{Z}$ & $\mathrm{P}$ & 13 \\
\hline Eunice pennata (Muller, 1776) & & & $\mathrm{C}, \mathrm{E}$ & & 2 \\
\hline Glycera capitata Orsted, 1843 & & & $\mathrm{C}, \mathrm{E}, \mathrm{B}^{\prime}$ & & 3 \\
\hline Harmothoe sp. & & & V & $\mathrm{X}$ & 2 \\
\hline Hermadion magalhaensi Kinberg, 1855 & I & $\mathrm{F}$ & $\mathrm{L}$ & $\mathrm{O}$ & 4 \\
\hline Hyalinoecia artifex Verrill, 1880 & & & & $\mathrm{X}, \mathrm{Y}$ & 2 \\
\hline Idanthyrsus macropaleus (Schmarda, 1861) & $\mathrm{S}$ & & $\mathrm{D}^{\prime}$ & & 2 \\
\hline Laetmonice producta Grube, 1877 & $A^{\prime}$ & & & & 1 \\
\hline Lanice sp. & & & E & & 1 \\
\hline Lumbrineridae unid. & & & $\mathrm{D}, \mathrm{N}, \mathrm{B}^{\prime}$ & & 3 \\
\hline Maldanidae unid. & & & $\mathrm{B}^{\prime}$ & & 1 \\
\hline Nephtys sp. & & F & & & 1 \\
\hline Nicolea chilensis (Schmarda, 1861) & $\mathrm{I}, \mathrm{R}, \mathrm{S}$ & $\mathrm{J}, \mathrm{K}, \mathrm{T}, \mathrm{U}$ & $\mathrm{E}, \mathrm{M}, \mathrm{N}, \mathrm{D}^{\prime}, \mathrm{Z}$ & W & 13 \\
\hline Nicon maculata Kinberg, 1866 & & & & $\mathrm{~B}, \mathrm{O}, \mathrm{A}, \mathrm{W}$ & 4 \\
\hline Oenonidae unid. & & & $\mathrm{D}, \mathrm{N}$ & & 2 \\
\hline Onuphis pseudoiridescens Averincev, 1972 & $A^{\prime}$ & & $\mathrm{B}^{\prime}$ & & 2 \\
\hline Paraonidae unid. & & & $\mathrm{B}^{\prime}$ & & 1 \\
\hline Phyllodocidae unid. & $\mathrm{A}^{\prime}$ & & $\mathrm{B}^{\prime}$ & & 2 \\
\hline Pista mirabilis Mdntosh, 1855 & & & $\mathrm{C}, \mathrm{D}^{\prime}, \mathrm{V}, \mathrm{B}^{\prime}$ & W, P & 6 \\
\hline Polyeunoa laevis Mclntosh, 1855 & $\mathrm{G}, \mathrm{H}, \mathrm{I}, \mathrm{Q}, \mathrm{S}, \mathrm{A}^{\prime}$ & $\mathrm{J}, \mathrm{T}, \mathrm{U}$ & $\begin{array}{l}\mathrm{C}, \mathrm{D}, \mathrm{M}, \mathrm{N}, \mathrm{L} \\
\mathrm{D}^{\prime}, \mathrm{V}, \mathrm{Z}, \mathrm{B}^{\prime}, \mathrm{C}^{\prime}\end{array}$ & $\mathrm{B}, \mathrm{A}, \mathrm{O}, \mathrm{W}, \mathrm{P}$ & 24 \\
\hline Polynoidae unid. & $\mathrm{A}^{\prime}$ & & $\mathrm{B}^{\prime}$ & Y & 3 \\
\hline Sabellidae 1 & $\mathrm{I}, \mathrm{A}^{\prime}$ & & $\mathrm{B}^{\prime}$ & & 3 \\
\hline Sabellidae 2 & I & & & & 1 \\
\hline Sabellidae Fabricinae & $\mathrm{Q}, \mathrm{R}, \mathrm{S}$ & $\mathrm{K}, \mathrm{T}, \mathrm{U}$ & E, D & $\mathrm{P}$ & 9 \\
\hline Serpula narconensis Baird, 1865 & $\mathrm{H}, \mathrm{I}, \mathrm{Q}, \mathrm{S}$ & $\mathrm{J}, \mathrm{F}, \mathrm{T}, \mathrm{U}$ & $\begin{array}{l}\mathrm{C}, \mathrm{E}, \mathrm{M}, \mathrm{L}, \mathrm{N} \\
\mathrm{Z}, \mathrm{B}^{\prime}, \mathrm{C}^{\prime}\end{array}$ & $\mathrm{O}, \mathrm{W}, \mathrm{P}$ & 19 \\
\hline Spionidae unid. & $\mathrm{H}, \mathrm{R}$ & & $\mathrm{E}$ & & 3 \\
\hline Syllidae unid. & $\mathrm{I}, \mathrm{A}^{\prime}$ & $\mathrm{J}$ & $\mathrm{B}^{\prime}$ & $\mathrm{O}$ & 5 \\
\hline Terebellidae unid. & Q & & & & 1 \\
\hline Terebellides malvinesis Bremec \& Elias, 1999 & & & $\mathrm{~B}^{\prime}$ & & 1 \\
\hline Thelepus sp. & & $\mathrm{J}, \mathrm{F}, \mathrm{T}, \mathrm{U}$ & $\mathrm{N}, \mathrm{V}$ & $\mathrm{O}, \mathrm{W}$ & 8 \\
\hline Travisia kerguelensis Mclntosh, 1855 & & & $\mathrm{~B}^{\prime}$ & & 1 \\
\hline Trypanosyllis gigantea Mdntosh, 1855 & I & $\mathrm{J}, \mathrm{U}$ & $\mathrm{C}, \mathrm{E}, \mathrm{N}, \mathrm{D}^{\prime}, \mathrm{V}, \mathrm{Z}$ & $\mathrm{P}$ & 10 \\
\hline
\end{tabular}


McIntosh, 1885, Eunereis patagonica (McIntosh, 1885) and Eucranta sp. were commonly registered within the MPA, although present once at the slope of the bank. Chaetopterus antarcticus Kinberg, 1866 (see Moore, Nishi, \& Rouse, 2017) was frequent in samples taken at the core zone of NMPA, where the total number of taxa was 22. The number of taxa was 13 and 32 at the buffer and transition zones of NMPA respectively. Samples from the slope included 14 polychaete taxa, with $H$. artifex and Nicon maculata Kinberg, 1866 as exclusive species. According to the current literature (Bremec \&
Giberto, 2008; Orensanz et al., 2012; Horton et al., 2018; OBIS, 2018), A. kerguelensis (ampharetid), H. artifex (onuphid), I. macropaleus (sabellariid), L. producta (aphroditid), $O$. pseudoiridescens (onuphid), P. mirabilis (terebellid) and T. malvinensis (trichobranchid) constitute new records for BB (Fig. 2).

MDS analysis among sites shows one main group of sampling stations from BB slope and the core, buffer and transition areas of NMPA (group 1), from which only two samples from the slope of the bank are excluded (31 and 33, group 2) (Table 1, Fig. 3A). The SIMPER test

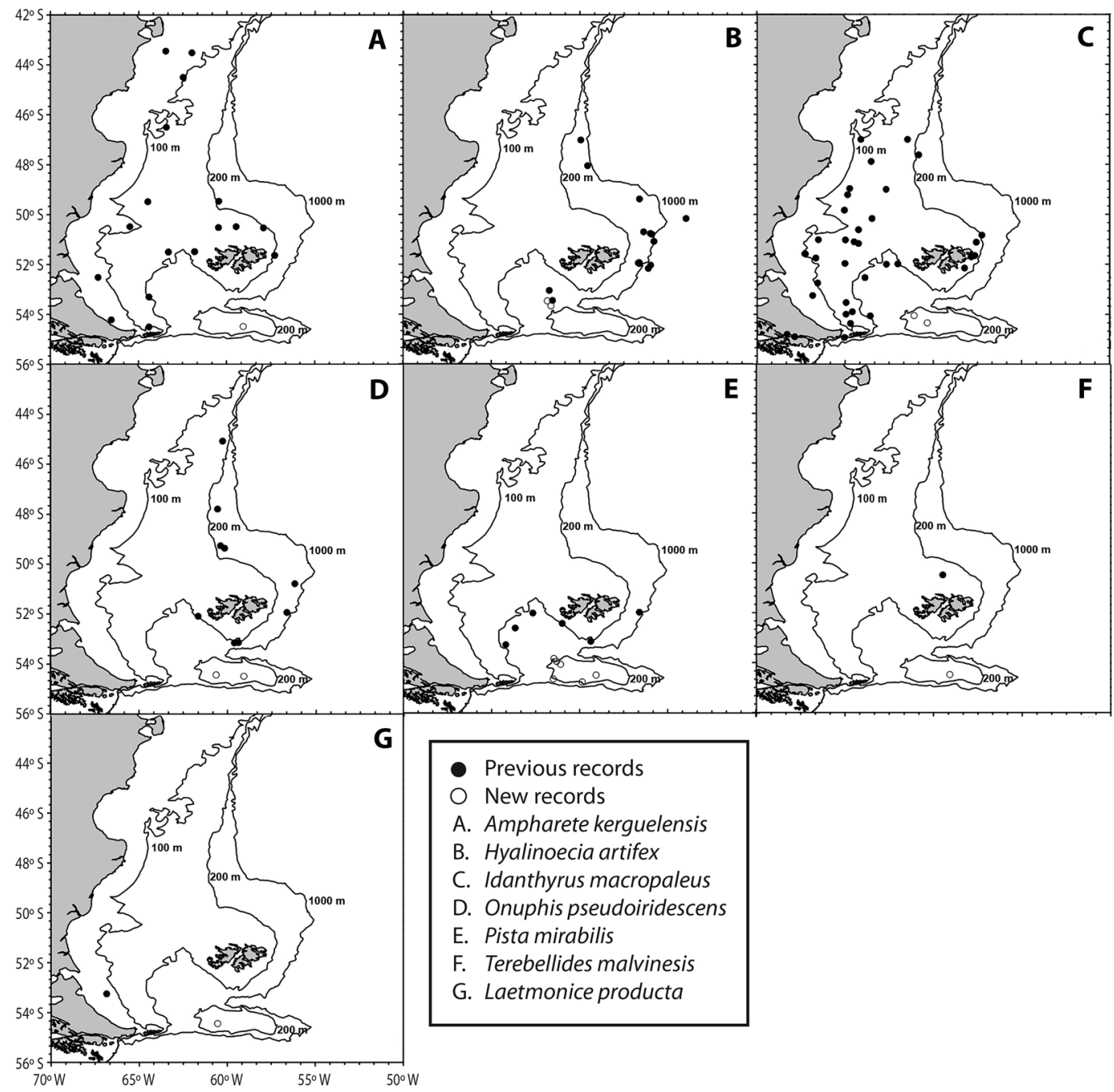

Fig. 2. Previous and new records of polychaete species for Burdwood Bank. 

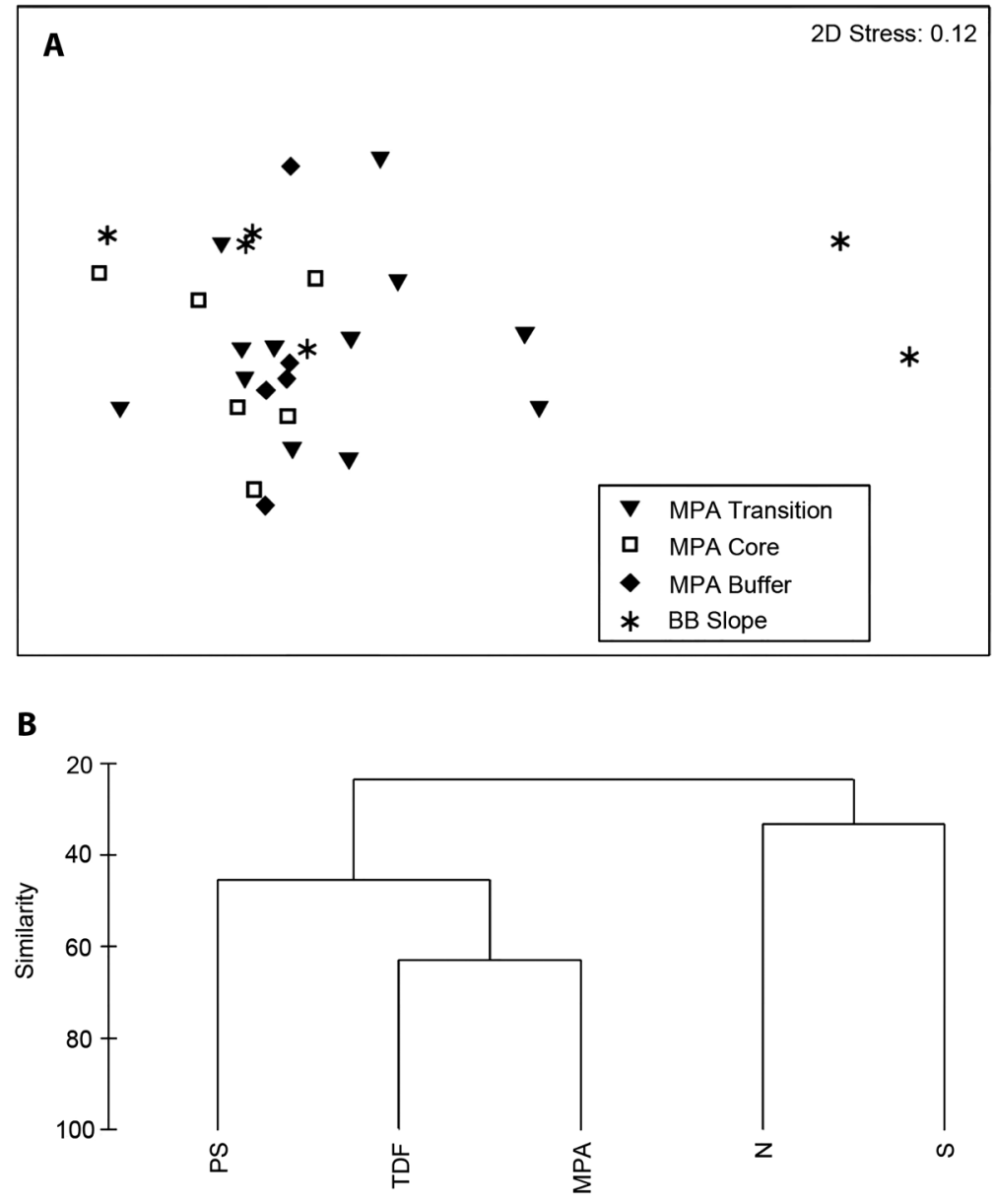

Fig. 3. A. Multidimensional Scaling among samples collected in the different zones of Namuncurá Marine Protected Area and Burdwood Bank slope during 2015, 2016 and 2017. B. Cluster analysis among polychaete data (presence-absence) from different Magellan areas: Patagonian Shelf (PS), Tierra del Fuego (TDF), Marine Protected Area (MPA), Burdwood Bank Neighboring Area (N) and Burdwood Bank Slope (S).

(presence-absence data) resulted in an average dissimilarity of $99.04 \%$ between both groups. P. laevis, S. narconensis, E. patagonica, $N$. chilensis, T. gigantea and Eucranta sp. contributed $82 \%$ to the average similarity $(0.32)$ of group 1. H. artifex contributed $100 \%$ to the average similarity $(0.50)$ of group 2 . The results of the ANOSIM between stations from NMPA and BB Slope (Global $\mathrm{R}=0.398 ; \mathrm{p}=$ $0.3 \%$ ) show no differences in the polychaetes species composition.

The regional comparison was performed with a data matrix of 82 samples from different
Magellan areas (Tables 1,2) and 73 polychaete taxa (Table 4). Cluster analysis (Fig. 3B) grouped NMPA-TDF (similarity $>60 \%$ ) and PS in group 1 ( $\sim 0.50$ similarity). Kinbergonuphis dorsalis (Ehlers, 1897), Aglaophamus virginis (Kinberg, 1866), A. kerguelensis, E. patagonica, S. narconensis, Travisia kerguelensis McIntosh, 1885 and P. laevis contributed $65 \%$ to the average similarity (0.15). Deep areas $\mathrm{S}$ and $\mathrm{N}$ clustered at lower similarity in group 2 (Fig. 3). P. laevis, S. narconensis, E. patagonica, Thelepus sp., H. artifex and N. maculata contributed $87 \%$ to the average 
TABLE 4

Polychaete taxa recorded at Magellan areas: Patagonian Shelf (PS), Tierra del Fuego (TDF), NMPA, BB Slope (BBS) and neighboring locations (N) during the cruises referred in Tables 1 and 2

\begin{tabular}{|c|c|c|c|c|c|}
\hline & PS & TDF & AMP & BBS & $\mathrm{N}$ \\
\hline Aglaophamus virginis (Kinberg, 1866) & $\mathrm{X}$ & $\mathrm{X}$ & $\mathrm{X}$ & & \\
\hline Ampharete kerguelensis McIntosh, 1885 & $\mathrm{X}$ & $\mathrm{X}$ & $\mathrm{X}$ & & \\
\hline Amphicteis gunneri antarctica Hessle, 1917 & $\mathrm{X}$ & & & & \\
\hline Anobothrus sp. & & & $\mathrm{X}$ & & \\
\hline Armandia sp. & $\mathrm{X}$ & & & & \\
\hline Boccardia sp. & & & $\mathrm{X}$ & & \\
\hline Chaetopterus antarcticus Kinberg, 1866 & $X$ & $\mathrm{X}$ & $\mathrm{X}$ & & \\
\hline Cistenides ehlersi (Hessle, 1917) & $X$ & $\mathrm{X}$ & & & \\
\hline Drilonereis tenuis (Ehlers, 1900) & $\mathrm{X}$ & & & & \\
\hline Eteone aurantiaca Schmarda, 1861 & $\mathrm{X}$ & & $\mathrm{X}$ & & \\
\hline Eucranta sp. & & & $\mathrm{X}$ & & \\
\hline Eunereis patagonica (McIntosh, 1885) & & $\mathrm{X}$ & $\mathrm{X}$ & $\mathrm{X}$ & $\mathrm{X}$ \\
\hline Eunice frauenfeldi Grube, 1866 & $\mathrm{X}$ & & & & \\
\hline Eunice pennata (Muller, 1776) & $\mathrm{X}$ & & & & \\
\hline Euphrosine armadilloides Ehlers, 1900 & $\mathrm{X}$ & & & & \\
\hline Euzonus sp. & $\mathrm{X}$ & & & & \\
\hline Glycera americana Leidy, 1855 & $\mathrm{X}$ & & & & \\
\hline Glycera capitata Orsted, 1843 & & $X$ & $X$ & & \\
\hline Glycera papillosa Grube, 1857 & $\mathrm{X}$ & $\mathrm{X}$ & $\mathrm{X}$ & & \\
\hline Glycinde sp. & $\mathrm{X}$ & & & & \\
\hline Harmothoe sp. & $\mathrm{X}$ & & $\mathrm{X}$ & $\mathrm{X}$ & \\
\hline Hemipodus sp. & $\mathrm{X}$ & & & & \\
\hline Hermadion magalhaensi Kinberg, 1855 & & & $\mathrm{X}$ & $\mathrm{X}$ & \\
\hline Hyalinoecia artifex Verril, 1880 & & & & $\mathrm{X}$ & \\
\hline Idanthyrsus macropaleus (Schmarda, 1861) & $X$ & $\mathrm{X}$ & $\mathrm{X}$ & & $\mathrm{X}$ \\
\hline Kinbergonuphis dorsalis (Ehlers, 1897) & $X$ & $\mathrm{X}$ & $X$ & & \\
\hline Laetmonice producta Grube, 1877 & & & $\mathrm{X}$ & & \\
\hline Lanice sp. & & & $\mathrm{X}$ & & \\
\hline Lumbriclymenella robusta Arwidsson, 1911 & $\mathrm{X}$ & & & & \\
\hline Lumbriclymeninae & $X$ & & & & \\
\hline Lumbrineriidae sp. 1 & & $\mathrm{X}$ & $\mathrm{X}$ & & \\
\hline Lumbrineriidae sp. 2 & & $\mathrm{X}$ & & & \\
\hline Lumbrineris cingulata Ehlers, 1897 & $X$ & & & & \\
\hline Magelonidae unid. & & $\mathrm{X}$ & & & \\
\hline Maldaniidae unid. & $\mathrm{X}$ & $\mathrm{X}$ & $\mathrm{X}$ & & \\
\hline Melinna cristata (M. Sars, 1851) & $\mathrm{X}$ & & & & \\
\hline Neanthes kerguelensis (McIntosh, 1885) & & $\mathrm{X}$ & & & \\
\hline Nephtys magellanica Augener, 1912 & $\mathrm{X}$ & & & & \\
\hline Nephtys sp. & $\mathrm{X}$ & & $\mathrm{X}$ & & \\
\hline Nereididae unid. & $\mathrm{X}$ & $\mathrm{X}$ & $\mathrm{X}$ & & \\
\hline Nicolea chilensis (Schmarda, 1861) & & & $\mathrm{X}$ & & \\
\hline Nicomache sp. & $X$ & & & & \\
\hline Nicon maculata Kinberg, 1866 & & & & $X$ & \\
\hline Nothria anoculata Orensanz, 1974 & $\mathrm{X}$ & & $\mathrm{X}$ & & \\
\hline Notocirrus lorum Ehlers, 1897 & $\mathrm{X}$ & $\mathrm{X}$ & & & \\
\hline
\end{tabular}


TABLE 4 (Continued)

\begin{tabular}{|c|c|c|c|c|c|}
\hline & PS & TDF & AMP & BBS & $\mathrm{N}$ \\
\hline Notocirrus virginis (Kinberg, 1865) & $\mathrm{X}$ & & & & \\
\hline Oenonidae unid. & & & $\mathrm{X}$ & & \\
\hline Onuphis pseudoiridescens Averincev, 1972 & & $\mathrm{X}$ & $\mathrm{X}$ & & \\
\hline Ophelina syringopyge (Ehlers, 1901) & $\mathrm{X}$ & & & & \\
\hline Orbiniidae unid. & $\mathrm{X}$ & $\mathrm{X}$ & & & \\
\hline Paraonidae unid. & & $\mathrm{X}$ & $\mathrm{X}$ & & \\
\hline Perkinsiana antarctica (Kinberg, 1867) & $\mathrm{X}$ & $\mathrm{X}$ & & & \\
\hline Phyllocomus crocea Grube, 1877 & & $\mathrm{X}$ & & & \\
\hline Phyllodoce patagonica (Kinberg, 1866) & $\mathrm{X}$ & & & & \\
\hline Phyllodocidae unid. & & $\mathrm{X}$ & $\mathrm{X}$ & & \\
\hline Phylo felix Kinberg, 1866 & $\mathrm{X}$ & $\mathrm{X}$ & & & \\
\hline Pista corrientis McIntosh, 1885 & $\mathrm{X}$ & & $\mathrm{X}$ & & \\
\hline Pista mirabilis McIntosh, 1885 & & $\mathrm{X}$ & $\mathrm{X}$ & $\mathrm{X}$ & \\
\hline Polyeunoa laevis McIntosh, 1885 & & $\mathrm{X}$ & $\mathrm{X}$ & $\mathrm{X}$ & $\mathrm{X}$ \\
\hline Polynoidae unid. & & $\mathrm{X}$ & $\mathrm{X}$ & $\mathrm{X}$ & $\mathrm{X}$ \\
\hline Potamilla antarctica (Kinberg, 1866) & $\mathrm{X}$ & & & & \\
\hline Sabellidae sp. 1 & $\mathrm{X}$ & $\mathrm{X}$ & $\mathrm{X}$ & & \\
\hline Sabellidae sp. 2 & & & $\mathrm{X}$ & & \\
\hline Sabellidae Fabricinae & & & $\mathrm{X}$ & $\mathrm{X}$ & \\
\hline Serpula narconensis Baird, 1865 & $\mathrm{X}$ & $\mathrm{X}$ & $\mathrm{X}$ & $\mathrm{X}$ & $\mathrm{X}$ \\
\hline Sphaerodoridae & $\mathrm{X}$ & & & & \\
\hline Spionidae unid. & & & $\mathrm{X}$ & & \\
\hline Syllidae unid. & $\mathrm{X}$ & $\mathrm{X}$ & $\mathrm{X}$ & $\mathrm{X}$ & \\
\hline Terebellidae unid. & & & $\mathrm{X}$ & & $\mathrm{X}$ \\
\hline Terebellides malvinensis Bremec \& Elias, 1999 & $\mathrm{X}$ & & $\mathrm{X}$ & & \\
\hline Thelepus sp. & $\mathrm{X}$ & $\mathrm{X}$ & $\mathrm{X}$ & $\mathrm{X}$ & $\mathrm{X}$ \\
\hline Travisia kerguelensis McIntosh, 1885 & $\mathrm{X}$ & $\mathrm{X}$ & $\mathrm{X}$ & & \\
\hline Trypanosyllis gigantea McIntosh, 1885 & & $\mathrm{X}$ & $\mathrm{X}$ & $\mathrm{X}$ & \\
\hline
\end{tabular}

similarity (0.21). The results of the ANOSIM developed to compare different Magellan areas does not show statistical differences among stations from NMPA, PS, TDF, S and N (Global R $=0.42 ; \mathrm{p}=0.1 \%$ ).

\section{DISCUSSION}

This research gives faunistic information on polychaetes from BB, including NMPA -a wide area delineated by the $200 \mathrm{~m}$ isobathand the slope of the bank. Data from recent monitoring cruises, particularly developed to conduct benthic sampling in the study area, have led to baseline knowledge about the invertebrate assemblages inhabiting a variety of substrates. A preliminary inventory of the most conspicuous benthic species collected during 2013 in NMPA and BB slope included nearly 250 taxa (Schejter et al., 2016), of which 19 were polychaetes mostly identified to family level. In the present study, a total of 39 polychaete taxa were identified, 20 of them to species level and previously recorded from other Subantarctic or Antarctic locations (Clarke \& Johnston, 2003; Orensanz et al., 2012; Horton et al., 2018; OBIS, 2018). However, some of them, collected in different zones of NMPA and/or BB slope (A. kerguelensis, H. artifex, I. macropaleus, L. producta, O. pseudoiridescens, P. mirabilis and T. malvinensis) constitute new records for $\mathrm{BB}$. Recent investigations showed that $\mathrm{BB}$ is home to more than 90 species of small organisms belonging to Peracarida (Doti 
et al., 2014; Chiesa et al., 2015), more than 280 species of small mollusks (Zelaya \& Guller, 2018) -most of them new records for the area or new species to science- and two new coral (Cairns, 2012) and sponge (Schejter, Bertolino et al., 2017) species. The bathymetric ranges of Asteroidea were extended for two species while the geographic distribution was updated for seven of them, being their first record in the NMPA (Fraysse et al., 2018). A new genus and species of cheilostome bryozoan showing an obligate association with a hermit crab was also described for the area (Lopez-Gappa, Liuzzi, \& Zelaya, 2017). It becomes clear that studies on polychaetes, and invertebrates in general, should continue in order to properly assess species richness in the MPA.

Some of the polychaete species were frequently collected and were distributed throughout the depth range $(91-785 \mathrm{~m})$ considered in this sampling (P. laevis, S. narconensis, E. patagonica, Thelepus sp.), while others were only collected within NMPA: $C$. antarcticus, $N$. chilensis, T. gigantea, Eucranta sp., Boccardia sp., Hermadion sp., Nephtys sp. and Sabellidae (two species). In fact, MDS shows a similar polychaete assemblage characterizing the study area (three zones of NMPA and slope locations), and $H$. artifex characterizing two locations at the NW deepest slope of the bank. Due to the sampling procedure, the taxa registered are mainly epibenthic or associated with other large colonial organisms collected in the area. In general, sponges were conspicuous components ( $40 \%$ to $88 \%$ of the total catch) in locations of the MPA, and cnidarians (mainly corals) were a dominant group in the catches at stations located at $>300$ $\mathrm{m}$ depth (Schejter, Genzano, Gaitán, Perez, \& Bremec, 2018a). The high diversity both of sponges (López Gappa \& Landoni, 2005; Schejter, Bertolino, Calcinai, Cerrano, \& Pansini, 2012) and cnidarians (López-González, Rodriguez, \& Vert, 2003; Margolin et al., 2014) in BB was previously reported. These sessile and three dimensional branched organisms are habitat-forming, as they provide substrate and refuge to a variety of associated species (see
Buhl-Mortensen et al., 2010). In this sampling, the most frequent free living polynoid P. laevis, nereidid E.patagonica and syllid T. gigantea, as well as the epibiotic serpulid $S$. narconensis, were mostly associated with octocorals or sponges. Ongoing studies registered 109 taxa, of which 19 were polychaete species, associated with octocorals (Thouarella sp., Dasystenella sp., Bayergorgia sp.) and hydroids (Amphisbetia sp., Sertularella sp., Symplectoscyphus sp., Grammaria sp., Abietinella sp., Halecium sp., Acryptolaria sp., Plumularia sp.) between 90-650 m depth in BB (Martin Sirito, 2019). P. laevis, widely distributed in the SW Atlantic (northwards up to Buenos Aires) and the Magellan, Sub-Antarctic and Antarctic regions, is often associated with corals (Barnich, Gambi, \& Fiege, 2012), which are also present and frequent beyond the shelf break of Argentina (Portela et al., 2012; Portela et al., 2015). Interspecific relationships between polychaetes and colonial hosts, like Cnidaria and Porifera, are numerous and include commensalism, endobiosis or epibiosis that facilitate, for instance, feeding strategies (Martin \& Britayev, 1998). Polychaetes can have a large variety of feeding modes (Fauchald \& Jumars, 1979); they were assumed to consume all suspended sources and the biofilm in a quantitative food-web analysis of a cold-water coral community in NE Atlantic, where biodiversity appears to be higher than in surrounding soft sediments (Oevelen et al., 2009). The nature and functionality of the associations between polychaetes and hosts are fields open to further research in Argentinean waters.

Other polychaete species were exclusively collected in particular locations of the study area. The parchment worm Chaetopterus antarcticus inhabited bottoms at nearly $100 \mathrm{~m}$ depth of NMPA, where Porifera and Bryozoa were conspicuous, together with other invertebrates (ophiuroids, serpulids, brachiopods, hydroids, peracarids, etc.) (Schejter, Genzano et al., 2018). These worms are active, mucusnet suspension feeders (Jumars, Dorgan, \& Lindsay, 2014), usually collected within their U-shaped tubes, typical of an infaunal habit 
(Fauchald \& Jumars, 1979; Rouse, 2001). This species is distributed throughout the shelf break frontal system along the $100 \mathrm{~m}$ isobath and up to $37{ }^{\circ} \mathrm{S}$ (Bremec \& Lasta, 2002) in habitats characterized by sandy soft-bottoms, like more than $90 \%$ of the Argentinean continental shelf (Parker, Paterlini, \& Violante, 1997), as well as in a submarine canyon located at $43^{\circ} 35^{\prime} \mathrm{S}$ - 59 $33^{\circ}$ ' W, 325 m depth (Bremec \& Schejter, 2010). On the other hand, deep locations at NW BB slope, characterized by muddy sediments and the presence of pennatulaceans and scleractinid corals (Schejter, Acuña, Garese, Cordeiro, \& Pérez, 2018), were inhabited by the onuphid species $H$. artifex. These tubiculous worms are carnivorous or carrion feeders and carry the tubes with them in a clearly discretely motile fashion, often in soft sediments (Jumars et al., 2014). Although information on the types of bottom at BB is still lacking (Falabella, 2018), the heterogeneity of the substrate and the patchiness of the benthic life in the study area appeared evident by means of underwater photographs acquired during a cruise in 2015 (Schejter, Martín, \& Lovrich, 2017). Ongoing research into the unknown bottom habitats will permit the appreciation of their true need for conservation.

The polychaete assemblages of BB (NMPA and slope) were compared with those of other Magellanic areas: Beagle Channel, Patagonian Shelf and three neighboring locations. The present analysis does not indicate statistical differences in the polychaete assemblage among locations, and species already known from the Magellanic Biogeographical Province (Orensanz et al., 2012; Bremec et al., 2000; Bremec et al., 2010), were registered at BB for the first time. In an extensive area of the Argentinean shelf between $39^{\circ} \mathrm{S}$ and $55^{\circ} \mathrm{S}$, in bottoms deeper than $100 \mathrm{~m}$, the most frequent species were $K$. dorsalis, I. macropaleus, $S$. narconensis, N. maculata, T. gigantean, E. patagonica and T. kerguelensis (Bremec et al., 2010). Sediment composition on the continental shelf is dominated by sands and silts of $<2$ mm grain size, at depths between 50 and 200 m (Bastida, Urien, Lichtschein, Roux, \& Arias,
1981; Bastida, Roux, \& Martinez, 1992). Other Magellanic habitats, like channels and fjords in the Pacific $\left(42^{\circ} \mathrm{S}-55^{\circ} \mathrm{S}\right)$ and the Straits of Magellan $\left(52^{\circ} \mathrm{S}-70^{\circ} \mathrm{W}\right)$, are heterogeneous and patchy with different types of sediments and consequently dominance and diversity of austral species is higher when compared with the homogeneous shelf habitats (Mariani et al., 1996; Gambi \& Mariani, 1999; Bremec et al., 2000; Montiel, Gerdes, Hilbig, et al., 2005). The polychaete assemblage registered at $\mathrm{BB}$ shows affinities with that distributed on the Argentinean continental shelf, on soft homogeneous bottoms. Although it is interesting to point out that most species are distributed in Antarctic waters (Clarke \& Johnston, 2003), much taxonomic work is needed to properly establish biogeographical connections. Souto (2014), after an exhaustive historical compilation and analysis of spatial distribution of polychaetes in Argentinean waters, found that Acrocirridae, Alciopidae, Ampharetidae, Arenicolidae, Capitellidae, Chrysopetalidae, Cossuridae, Euphrosinidae, Flabelligeridae, Polynoidae, Maldanidae and Phyllodocidae, among others, have been scarcely registered due to poor sampling coverage. It must be pointed out that high endemism in the distribution of species was showed in a recent revision on austral orbiniids (Blake, 2017).

The present results indicate strong connections between the fauna collected at NMPA and the polychaete assemblages of other Magellan areas dominated by soft bottoms. However, deeper slope areas of BB are inhabited by large species (i.e. H. artifex) not registered in the NMPA. Moreover, the slope area of BB was recently highlighted because cold-water coral ecosystems were recorded in several sites, meeting the characteristics of Vulnerable Marine Ecosystems (VME) (Schejter, Genzano, et al., 2018), which must be protected from fishing gears that destroy seafloor habitat (see Hall, 1999). The biogeographic importance of the $\mathrm{BB}$ as connection for benthic polychaete species between South America and the Antarctic Peninsula merits future investigation. Trophic relationships and the role of epibiotic 
relationships in enhancing biodiversity in BB seabeds should also be explored.

Ethical statement: authors declare that they all agree with this publication and made significant contributions; that there is no conflict of interest of any kind; and that we followed all pertinent ethical and legal procedures and requirements. All financial sources are fully and clearly stated in the acknowledgements section. A signed document has been filed in the journal archives.

\section{ACKNOWLEDGEMENTS}

We thank Jonathan Flores, Esteban Gaitán, Agustín Garese, Ricardo Gonzalez, Marina Güller and Diego Zelaya, for help on board the RV "Puerto Deseado" during sampling procedures and samples from "Pr. García". We are also deeply grateful to scientific and navy crews of the cruises. Anonymous reviewers improved the manuscript. This is INIDEP Contribution $\mathrm{N}^{\mathrm{o}}$ 2168. This is Marine Protected Area Namuncurá (National Law 26.875, Argentina) Contribution $\mathrm{N}^{\circ} 19$.

\section{RESUMEN}

\section{Poliquetos del Banco Burdwood: \\ Área Marina Protegida "Namuncurá I" y Talud. SO del Oceano Atlántico}

Introducción: La primera Área Marina Protegida de mar abierto (no costera) en Argentina, llamada "Namuncurá I" (AMPN), fue creada en 2013 en el Banco Burdwood (BB), una plataforma sumergida ubicada unos $200 \mathrm{~km}$ al sur de las Islas Malvinas y $150 \mathrm{~km}$ al Este de la Isla de los Estados. En el Océano Atlántico SO. El área comprende tres diferentes áreas de manejo: la central ("el núcleo", con protección estricta, solo Control y actividades de monitoreo), rodeada por un área "de amortiguación" (solo actividades autorizadas, como investigación científica) y un área externa de "transición" (se contemplan actividades productivas y extractivas en el Plan de Manejo). Más allá del área de transición, el talud y las áreas adyacentes no presentan medidas de protección. Objetivos: proporcionar un inventario de poliquetos colectados durante 2016 y 2017 en el Núcleo (98 m de profundidad), de Amortiguación (128 m), de Transición (133 m-189 m) y Talud (220 m-798 m). Métodos: las muestras fueron obtenidas con una red de arrastre para caracterizar las asociaciones faunísticas y para compararlas con otras áreas Magallánicas. Análisis de Escalamiento Multidimensional y de agrupamiento aglomerativo jerárquico fueron aplicados a una matriz de similaridad de Bray-Curtis para determinar las asociaciones de poliquetos en el AMP - Talud del BB y región Magallánica, respectivamente. Resultados: Un total de 918 individuos, que corresponden a 39 taxones distribuidos en 22 familias, fueron registrados en las muestras del AMPN y talud del BB, mayormente epibentónicos o asociados a grandes corales. Ampharete kerguelensis McIntosh, 1885; Hyalinoecia artifex Verrill, 1880; Idanthyrsus macropaleus (Schmarda, 1861); Laetmonice producta Grube, 1877; Onuphis pseudoiridescens Averincev, 1972; Pista mirabilis McIntosh 1885 y Terebellides malvinensis Bremec \& Elias, 1999 constituyen nuevos registros para el Banco Burdwood. Un ensamble de poliquetos similar caracteriza a las tres áreas del AMPN y talud, mientras que H. artifex caracteriza los sitios más profundos del talud del banco. Conclusiones: Estos resultados indican una fuerte conexión entre la fauna colectada en el NMPA y los ensambles de poliquetos de sustratos blandos de otras áreas Magallánicas. La importancia biogeográfica del BB como una conexión entre Sudamérica y la Península Antártica justifican futuras investigaciones.

Palabras clave: Nueva Área Marina Protegida, Banco Burdwood, Poliquetos, Océano Atlántico Sudoccidental.

\section{REFERENCES}

Arntz, W., Lovrich, G. A., \& Thatje, S. (Eds.). (2005). The Magellan-Antarctic connection: links and frontiers at high southern latitudes. Scientia Marina, 69, (Suppl. 2), 373 .

Barnich, R., Gambi, M. C., \& Fiege, D. (2012). Revision of the genus Polyeunoa McIntosh, 1885 (Polychaeta, Polynoidae). Zootaxa, 3523, 25-38.

Barnich, R., Orensanz, J., \& Fiege, D. (2012). Remarks on some scale worms (Polychaeta, Polynoidae) from the Southwest Atlantic with notes on the genus Eucranta Malmgren, 1866, and description of a new Harmothoe species. Marine Biodiversity, 42,(3), 395-410.

Bastida, R., Roux, A., \& Martínez, D. (1992). Benthic communities of the Argentine continental shelf. Oceanologica Acta, 15(6), 687-698.

Bastida, R., Urien, C., Lichtschein, V., Roux, A., \& Arias, P. (1981). Investigación sobre comunidades bentónicas. Características generales del sustrato (Campañas IV, V, X y XI del B/I "Shinkai Maru"). Contribuciones Instituto Nacional Investigación y Desarrollo Pesquero, 383, 318-339.

Blake, J. (1983). Polychaetes of the Family Spionidae from South America, Antarctica, and adjacent seas and islands. Antarctic Research Series, 29, 205-288. 
Blake, J. (2017). Polychaeta Orbiniidae from Antarctica, the Southern Ocean, the Abyssal Pacific Ocean, and off South America. Zootaxa, 4218(1), 001-145.

Böggemann, M., \& Orensanz, J. M. (2007). Glyceriformia Fauchald, 1977 (Annelida: "Polychaeta") from the SW Atlantic Shelf, between $30^{\circ}$ and $45^{\circ}$ S. Mitteilungen aus dem Hamburgischen Zoologischen Museum und Institut, 104, 11-59.

Bremec, C., \& Giberto, D. 2008. Poliquetos. In D. Boltovskoy (Ed.). Atlas de sensibilidad ambiental de la Costa y el Mar Argentino. Proyecto "Prevención de la Contaminación Costera y Gestión de la Diversidad Biológica Marina" (GEF, BM, PNUD, SAyDS), componente "Conservación de la Diversidad Biológica y Prevención de la Contaminación Marina en Patagonia" (Proy. ARG 02/018). Retrieved from www. ambiente.gov.ar.

Bremec, C., \& Elias, R. (1999). Terebellides species (Polychaeta, Trichobranchidae) in cold-temperate waters from Southwestern Atlantic Ocean. Ophelia, $3,177-186$

Bremec, C., \& Lasta, M. (2002). Epibenthic assemblage associated with scallop (Zygochlamys patagonica) beds in the Argentinian shelf. Bulletin of Marine Science, 70, (1), 89-105.

Bremec, C., \& Schejter, L. (2010). Benthic diversity in a submarine canyon in the Argentine sea. Revista Chilena de Historia Natural, 83, 453-7.

Bremec, C., Elias, R., \& Gambi, M. C. (2000). Comparison of polychaete community composition from the patagonian shelf and Strait of Magellan, preliminary results from cruises "Shinkai Maru" IV, V, X and XI (1978-1979) and 2nd. P.N.R.A. Oceanographic Cruise (1991). Bulletin of Marine Science, 67(1), 189-197.

Bremec, C., Souto, V., \& Genzano, G. (2010). Polychaete assemblages in SW Atlantic: Results of "Shinkai Maru" IV, V, X and XI (1978-1979) cruises in Patagonia and Buenos Aires. Anales Instituto de la Patagonia, 38(2), 47-57.

Buhl-Mortensen, L., Vanreusel, A., Gooday, A. J., Levin, L. A., Priede, I. G., Buhl-Mortensen, P., ...Raes, M. (2010). Biological structures as a source of habitat heterogeneity and biodiversity on the deep ocean margins. Marine Ecology, 31, 21-50.

Cairns, S. D. (2012). New primnoid genus and species (Alcyonacea: Primnoidae) from the southwestern Atlantic. Proceedings of the Biological Society of Washington, 125, 180-8.

Cañete, J., Leighton, G., \& Aguilera, F. (1999). Polychaeta from Aysén Fjord, Chile: distribution, abundance and biogeographical comparison with the shallow soft-bottom polychaete fauna from Antarctica and the Magellan Province. Scientia Marina, 63, (Suppl 1), 243-252.

Chiesa, I., Urteaga, D., Martinez, A. I., Doti, B. L., \& Roccatagliata, D. (2015). Biodiversidad de anfípodos bentónicos del AMP Namuncurá -Banco Burdwood. In L. Riccialdelli, M. P. Sotelano \& C. C. Boy (Eds.) Libro de Resúmenes IX Jornadas Nacionales de Ciencias del Mar y XVII Coloquio

de Oceanografia : Ciencia y Sociedad: Integrando saberes en los estudios del mar (p. 263). Ushuaia, Argentina: Centro Austral de Investigaciones Científicas (CADIC).

Clarke, K. R., \& Gorley, R. N. (2006). PRIMER v6: UserManual/Tutorial.PRIMER-ELtd. Plymouth, UK: Plymouth Marine Laboratory,

Clarke, A., \& Johnston, N. M. (2003). Antarctic marine benthic diversity. Oceanography Marine Biology, $41,47-114$

Doti, B., Chiesa, I., Alberico, N., Sganga, D., Giachetti, C., Pereira, E., \& Roccatagliata, D. (2014). Biodiversidad de crustáceos Peracarida del banco Burdwood/ Namuncurá: resultados preliminares. In G. Pereira \& R. Maneyro (Eds.) Tercer Congreso Uruguayo de Zoología "Prof. Dr. Raúl Vaz - Ferreira" (pp. 191192). Montevideo, Uruguay: Facultad de Ciencias, Universidad de la República.

Elías, R., Bremec, C., Lana, P. C., \& Orensanz, J. M. (2003). Opheliidae (Polychaeta) from the southwestern Atlantic Ocean. Hydrobiologia, 496, 75-85.

Falabella, V. (Ed.). 2018. Área Marina Protegida Namuncurá - Banco Burdwood. Contribuciones para la línea de base y el plan de manejo. Buenos Aires, Argentina: Jefatura de Gabinete de Ministros.

Fauchald, K., \& Jumars, P. A. (1979). The diet of worms: A study of polychaete feeding guilds. Oceanography Marine Biology Annual Review, 39, 193-284.

Findlay, A. G. (1867). A sailing directory for the Ethiopic or South Atlantic Ocean including the coasts of South America and South Africa (5 ed.), London, UK:. RH Laurie.

Fraysse, C., Calcagno, J., \& Pérez, A. (2018). Asteroidea of the southern tip of South America, including Namuncurá Marine Protected Area at Burdwood Bank and Tierra del Fuego Province, Argentina. Polar Biology, 41, 2423-2433. https://doi.org/10.1007/ s00300-018-2377-3.

Gambi, M. C., \& Mariani, S. (1999). Polychaetes of the soft bottoms of the Straits of Magellan collected during the Italian oceanographic cruise in FebruaryMarch 1991. Scientia Marina, 63(Suppl. 1), 233-242. 
Griffiths, H. J., Linse, K., \& Barnes, D. K. A. (2008) Distribution of macrobenthic taxa across the Scotia Arc, Southern Ocean. Antarctic Science, 20, 213-26.

Guerrero, R. A., Baldoni, A., \& Benavides, H. (1999). Oceanographic conditions at the southern 365 end of the Argentine continental slope. INIDEP Scientific Documents, 5, 7-22.

Güller, M., Abelando, M., Urcola, M., \& Zelaya, D. (2015). Invertebrados bentónicos: estudios faunísticos en la primera área marina protegida oceánica de argentina. Informe de Campaña "GC Pr. García 2015”. Buenos Aires, Argentina: Universidad de Buenos Aires.

Hall, S. (1999). The effects of fishing on marine ecosystems and communities. Oxford, UK: Blackwell.

Hartman, O. (1953). Non-pelagic polychaetes of the Swedish Antarctic Expedition. 1901-1903. Further Zoological Results of the Swedish Antarctic Expedition, 4(11), 1-83.

Hartman, O. 1966. Polychaeta Myzostomidae and Sedentaria of Antarctica (Antarctic Research Series, 7). Washington, D.C.: American Geophysical Union.

Hartmann-Schroeder, G. (1983). Die Polychaeten der 15., 36. und 76. Reise von FFS "Walther Herwig" zum patagonischen Schelf (Südwest-Atlantik). Senckenbergiana maritima, 15(4/6), 251-277.

Hartmann-Schroeder, G., \& Hartmann, G. (1962). Zur Kenntnis des Eulitorals der chilenischen Pazifikküste und der argentinischen Küste Südpatagoniens unter besonderer Berücksichtigung der Polychaeten und Ostracoden. Hamburg, Germany: Zoologisches Staatsinstitut und Zoologisches Museum,

Horton, T., Kroh, A., Ahyong, S., Bailly, N., Boyko, C. B., Brandão, S. N., ... Zhao, Z. (2018). World Register of Marine Species. Retrieved from http://www.marinespecies.org at VLIZ. doi:10.14284/170

Jumars, P. A., Dorgan, K., \& Lindsay, S. (2014). Diet of Worms Emended: An Update of Polychaete Feeding Guilds. A Family-by-Family Updates Supplemental Material. Annual Review Marine Science, 7, 497-520.

Lana, P. C., \& Bremec, C. (1994). Sabellariidae (Annelida, Polychaeta) from South America. Mémoires Museum Nationale Histoire Naturelle, 162, 209-221.

López Gappa, J., \& Landoni, N. (2005). Biodiversity of Porifera in the Southwest Atlantic between $35^{\circ} \mathrm{S}$ and $56^{\circ}$ S. Revista Museo Argentino Ciencias Naturales, 7, 191-219.

López Gappa, J., Liuzzi, M, \& Zelaya, D. (2017). A new genus and species of cheilostome bryozoan associated with hermit crabs in the subantarctic Southwest Atlantic. Polar Biology, 41(4), 733-741. https://doi. org/10.1007/s00300-017-2234-9.
López-González, P., Rodríguez, E., \& Vert, N. (2003). Biogeography and Ecology of Cnidaria. Berichte zur Polar- und Meeresforschung, 462, 13-18.

Lutz, V. A., Segura, V., Dogliotti, A. I., Gagliardini, D. A., Bianchi, A. A., \& Balestrini, C. F. (2010). Primary production in the Argentine Sea during spring estimated by field and satellite 381 models. Journal of Plankton Research, 32(2), 181-195.

Margolin, A. R., Robinson, L. F., Burke, A., Waller, R. G., Scanlon, K. M., Roberts, M. L., Auro, M. E., \& van de Flierdt, T. (2014). Temporal and spatial distributions of cold-water corals in the 384 Drake Passage: insights from the last 35,000 years. Deep Sea Research II, 99, 237-48.

Mariani, S., Gambi, M. C., Lorenti, M., \& Mazzella. L. (1996). Benthic populations of the soft bottoms in the Strait of Magellan (Southern America): biodiversity, distribution and biogeography of polychaetes and crustacean isopods. Biologia Marina Mediterranea, 3(1), 155-158.

Martin Sirito, S. (2019). Fauna asociada a corales (Octocarallia) e hidroides (Hydrozoa) del Área Marina Protegida "Namuncurá" (Banco Burdwood) y zonas profundas adyacentes (Tesis de Licenciatura). Mar del Plata, Argentina: Universidad Nacional de Mar del Plata.

Martin, D. \& Britayev, T. A. (1998). Symbiotic polychaetes: Review of known species. Oceanography and Marine Biology: An Annual Review, 36, 217-340.

Montiel, A., Gerdes, D., \& Arntz, W. (2005). Distributional patterns of shallow-water polychaetes in the Magellan region: a zoogeographical and ecological sinopsis. Scientia Marina, 69(Suppl. 2), 123-133.

Montiel, A., Gerdes, D., Hilbig, B., \& Arntz, W.E. (2005). Polychaete assemblages on the Magellan and Weddell Sea shelves: comparative ecological evaluation. Marine Ecology Progress Series, 297, 189-202.

Montiel, A., Quiroga, E., \& Gerdes, D. (2011). Diversity and spatial distribution patterns of polychaete assemblages in the Paso Ancho, Straits of Magellan, Chile. Continental Shelf Research, 31, 304-314.

Montiel, A., Tarazona, J., Roux, A., Vera, G., Quipuzcoa, L., \& Enriquez, E. (2003). Biogeography and Ecology of Polychaeta. Berichte zur Polar- und Meeresforschung, 462, 29-33.

Moore, J., Nishi, E., \& Rouse, G. (2017). Phylogenetic analysis of Chaetopteridae (Annelida). Zoologica Scripta, 46, 596-610.

Ocean biogeographic information system (2018). Ocean biogeographic information system. Retrieved from https://obis.org.. 
Oevelen, D., Duineveld, G., Lavaleye, M., Mienis, F., Soetaert, K., \& Heip, C. (2009). The cold-water coral community as a hot spot for carbon cycling on continental margins: A food-web analysis from Rockall Bank (northeast Atlantic). Limnology Oceanography, 54(6), 1829-1844.

Orensanz, J. M. (1974). Los Anélidos Poliquetos de la Provincia Biogeográfica Magallánica. I. Catálogo de las Especies Citadas hasta 1974 (Contribución Técnica 1). Santa Clara del Mar, Argentina: Laboratorio de Comunidades Bentónicas.

Orensanz, J. M. (1990). The Eunicemorph polychaete annelids from Antarctic and Subantarctic Seas. Biology of the Antarctic Seas XXI. Antarctic Research Series, 52, 1-183.

Orensanz, J., Diez, M., Ferrando, A. \& Trovant, B. (2012). Unpublished data. Polychaete Annelids from the Southwestern Atlantic and its adjacencies. An Annotated Catalogue of Records. Puerto Madryn, Chubut, Argentina: Centro Nacional Patagónico (CENPAT/ CONICET).

Parker, G., Paterlini, M. C., \& Violante, R. A. (1997). The sea floor. In E. E. Boschi (Ed.). El Mar Argentino y sus Recursos Pesqueros (pp. 65-87). Mar del Plata, Argentina: INIDEP.

Piola, A. R., \& Gordon, A. L. (1989) Intermediate waters in the southwest South Atlantic. Deep-Sea Research, $36,1-16$.

Portela, J., Acosta, J., Cristobo, J., Muñoz, A., Parra, S., Ibarrola, T., ... Marin, P. (2012). Management Strategies to Limit the Impact of Bottom Trawling on VMEs in the High Seas of the SW Atlantic. In A. Cruzado (Ed.), Marine Ecosystem (pp. 199-228). London, UK: InTechOpen.

Portela, J., Cristobo, J., Ríos, P., Acosta, J., Parra, S., del Río, J. L., ... Marin, P. (2015). A first approach to assess the impact of bottom trawling over Vulnerable Marine Ecosystems on the high seas of the Southwest Atlantic. In Y. H. Lo, J. A. Blanco, \& S. Roy (Eds.). Biodiversity in Ecosystems, linking structure and function (pp. 271-298), London, UK: InTechOpen.

Rouse, G. W. (2001). Chaetopteridae. In G. Rouse, \& F. Pleijel (Eds.). Polychaetes (pp. 256-260). New York, EEUU: Oxford University Press.

Rozbaczylo, N., Ríos, C., \& Mutschke, E. (1997). Poliquetos de la región de Magallanes: estado actual de su conocimiento a través de un análisis histórico y un estudio de caso. In Universidad de Magallanes (Ed.). Resúmenes del Seminario-Taller Internacional sobre Investigación Biológica Marina en el área de Magallanes en relación con la Antártida, Univ. de Magallanes, Punta Arenas, Chile, 7-11 de abril, 1997. Punta Arenas, Chile: Universidad de Magallanes.

Sanfilippo, R. (1994). Polychaete distribution patterns on Chlamys patagonica of the Magellan Strait. In J. C. Dauvin, I. Laubier, \& D. J Reish (Eds.). Actes de la 4ème Confèrence Internationale des Polychètes Angers France 27th. July $2^{\text {nd. }}$ August 1992. Mémoires du Musséum National d'Histoire Naturelle 162 (535540 pp.). Paris, France: Éditions du Muséum.

Schejter, L., Bertolino, M., \& Calcinai, B. (2017). Description of Antho (Plocamia) bremecae sp. nov. and checklist of Microcionidae (Demospongiae: Poecilosclerida) from Burdwood Bank and neighboring areas, SW Atlantic Ocean. Zootaxa, 4312(3), 580-594.

Schejter, L., Martín, J., \& Lovrich, G. (2017). Unveiling the submarine landscape of the Namuncurá Marine Protected 373 Area, Burdwood Bank, SW Atlantic Ocean. Pan-American Journal of Aquatic Sciences, 12(3), 248-253.

Schejter, L., Acuña, F., Garese, A., Cordeiro, R., \& Pérez, C. (2018). Sea Pens (Cnidaria: Pennatulacea) from Argentine waters: new distributional records and first report of associated anemones. Pan-American Journal of Aquatic Sciences, 13(4), 292-301.

Schejter, L., Bertolino, M., Calcinai, B., Cerrano, C., \& Pansini, M. (2012). Banco Burdwood: resultados preliminares sobre composición y riqueza específica de esponjas (Phylum Porifera), a partir de muestras colectadas en la campaña del buque rompehielos estadounidense "Nathaniel B. Palmer", abril-mayo 2008 (Research Report, 122). Mar del Plata, Argentina: INIDEP.

Schejter, L., Genzano, G., Gaitán, E., Perez, C., \& Bremec, C. (2018). Bosques animales marinos en el Atlántico Sudoccidental: el Banco Burdwood, $54^{\circ} \mathrm{S}$ (Research Report, 38). Mar del Plata, Argentina: INIDEP.

Schejter, L., Rimondino, C., Chiesa, I., Diaz de Astarloa, J. M., Doti, B. L., Elias, R., ... Bremec, C. S. (2016). Namuncura Marine Protected Area: an oceanic hot spot of benthic biodiversity at Burdwood Bank, Argentina. Polar Biology, 39, 2373-2386.

Souto, V. (2014). Invertebrados bentónicos en el Mar Argentino: estado actual del conocimiento, riqueza especifica y patrones de distribución (Tesis Doctoral). Mar del Plata, Argentina: Universidad Nacional de Mar del Plata. 
Thomson, M. R. A. (2004). Geological and palaeoenvironmental history of the Scotia Sea Region as a basis for biological interpretation. Deep Sea Research - Part II, 51, 1467-1487.

Uschakov, P. V. (1962). Polychaetous Annelids of the Families Phyllodocidae and Aphroditidae from the Antarctic and Subantarctic. Biological Results of the Soviet Antarctic Expedition 1955-1958, Exploration of the Fauna of the Seas I, X, 129-189.
Wesenberg-Lund, E. (1962). Polychaeta Errantia. Reports Lund University Chile Expedition 1948-49. Lunds Universitets Arsskrift, 57 (12), 1-130.

Zelaya, D., \& Güller, M. (2018). Documentando la diversidad biológica del Área Marina Protegida Namuncurá/ Banco Burdwood: ¿qué nos dicen los moluscos? $X$ Jornadas Nacionales de Ciencias del Mar. Buenos Aires, Argentina: Universidad de Buenos Aires. 